\title{
Agreement between quantitative and qualitative sensory testing of changes in orofacial somatosensory sensitivity.
}

\begin{tabular}{|c|c|}
\hline Journal: & Journal of Oral Rehabilitation \\
\hline Manuscript ID & JOR-16-0266.R1 \\
\hline Manuscript Type: & Original Article \\
\hline Date Submitted by the Author: & $\mathrm{n} / \mathrm{a}$ \\
\hline Complete List of Authors: & $\begin{array}{l}\text { Agbaje, Jimoh; Katholieke Universiteit Leuven, OMFS-IMPATH Research } \\
\text { Group, Department of Imaging and Pathology, } \\
\text { De Laat, Antoon; Catholic University of Leuven, Oral and Maxillofacial } \\
\text { Surgery, School of Dentistry, Oral Pathology and Maxillo-Facial Surgery } \\
\text { constantinus, Politis; KULeuven, Oral and maxillofacial surgery } \\
\text { Svensson, Peter; Aarhus University, section of Orofacial Pain and Jaw } \\
\text { Function } \\
\text { Baad-Hansen, Lene; Aarhus University, section of Orofacial Pain and Jaw } \\
\text { Function }\end{array}$ \\
\hline Patient: & adult, adolescent \\
\hline Condition: & neurosensory functioning \\
\hline Co-morbidity: & Not applicable \\
\hline Content method: & research, pain management \\
\hline Study method: & clinical research \\
\hline
\end{tabular}




\section{Agreement between quantitative and qualitative sensory testing of changes in orofacial somatosensory sensitivity.}

Jimoh Agbaje ${ }^{1 *}$, Antoon De Laat ${ }^{2}$, Constantinus Politis ${ }^{1,3}$, Peter Svensson $^{4,5}$, Lene Baad-Hansen ${ }^{4}$

${ }^{1}$ OMFS-IMPATH Research Group, Department of Imaging and Pathology, KU Leuven, Leuven, Belgium

${ }^{2}$ Department of Oral Health Sciences, KU Leuven, Leuven, Belgium

${ }^{3}$ Oral and Maxillofacial Surgery, Leuven University Hospitals, Belgium

${ }^{4}$ Section of Orofacial Pain and Jaw Function, Department of Dentistry, Aarhus University, Aarhus, Denmark and Scandinavian Center for Orofacial Neurosciences (SCON)

${ }^{5}$ Department of Dental Medicine, Karolinska Institutet, Huddinge, Sweden

* Corresponding author:

Jimoh Agbaje

OMFS-IMPATH Research Group, Department of Imaging and Pathology, Faculty of Medicine, Catholic University Leuven, Kapucijnenvoer 33, 3000 Belgium

Business telephone number: +32 16341963

Fax: +32 16332437

Email: joagbaje@gmail.com 
Abstract

Qualitative somatosensory testing (QualST) is a simple chairside test. It can be used to roughly assess the presence or absence of altered somatosensory function. To use QualST clinically, it is important to assess its agreement with quantitative sensory testing (QST). The aims of this study were to assess the agreement between QST and QualST when testing the modulation of facial sensitivity by capsaicin in healthy participants and to explore the agreement between QST and QualST in assessing the intraoral sensory function in clinical atypical odontalgia (AO) patients. Eighteen healthy pain-free adults and data from $27 \mathrm{AO}$ patients were included in the study.

Thirteen QST and three QualST parameters were evaluated at each site. Z-scores were computed for healthy participants and LossGain scores were created. The agreement observed between QST and QualST in participants with no alterations in facial sensation (placebo) was good, i.e. ranging from $89 \%$ to $94 \%$. A poorer agreement was seen after capsaicin application in all test modalities with agreement ranging from $50 \%$ to $72 \%$. The commonest misclassification observed was participants classified as normal according to QST, but hyper- or hyposensitive according to QualST after capsaicin application, especially for cold and pinprick. A similar trend was observed in AO patients where patients classified as normal using QST were misclassified as hypersensitive and in few patients as hyposensitive by QualST. In conclusion, the study showed that QualST may be used as a screening tool in the clinical setting, especially to show that subjects have normal sensory function.

Key words

capsaicin; placebo; somatosensory profiling; quantitative sensory testing, qualitative sensory testing; atypical odontalgia 


\section{Background}

Nerve damage and altered sensation are common complications of orofacial trauma and some oral and maxillofacial treatments ${ }^{1-3}$. The resulting effect can range from mild complications such as transient hypoesthesia to life changing effects such as neuropathic pain ${ }^{4-7}$. Clinical symptoms of nerve damage such as altered sensations, e.g. somatosensory impairment, allodynia, persistent pain, pain and discomfort in the orofacial region, usually interfere negatively with daily activities ${ }^{8}$. Persistent uncontrollable postoperative pain and neuropathic pain are disabling iatrogenic conditions with severe medical, economic and psychological implications ${ }^{9-11}$. Early detection of nerve damage may aid treatment planning of nerve repair and lead to a reduction of the sensory impairment ${ }^{6,12-16}$.

A standardized battery of quantitative sensory testing (QST) was developed in 2006 by the German Research Network on Neuropathic Pain (Deutscher Forschungsverbund Neuropathischer Schmerz (DFNS)) to study somatosensory function ${ }^{17}$. This protocol has been proven to be sufficiently reliable for evaluation of gain or loss of somatosensory function at several body sites including the orofacial region ${ }^{18-21}$. The somatosensory profiles obtained from QST may be used to explore mechanisms behind different pain conditions but with regards to neuropathic pain, somatosensory profiles may vary quite a lot even within the same condition ${ }^{20,22}$.

Though QST is sufficiently reliable, its application in the clinical setting is limited as a result of a need for highly trained personnel required to operate the required equipment as well as the amount of time needed to complete the full battery of tests ${ }^{23}$. Therefore, a simpler and more qualitative clinical method to assess somatosensory testing has been advocated by some authors ${ }^{23-26}$. Qualitative somatosensory testing (QualST) is an example of such a simple chairside test ${ }^{23,26}$. It can be used to roughly assess the presence or not of altered somatosensory function. It is simple to apply and no extensive training and expensive equipment are needed. The test takes less than 5 min to perform but it provides information about crude hyposensitivity or hypersensitivity to touch, cold and pinprick stimuli. Though other stimulus modalities exist ${ }^{27}$ these modalities cover the function of both $\mathrm{A} \beta-, \mathrm{A} \delta$ and $\mathrm{C}$-fibers ${ }^{23}$. QualST has been tested intraorally with promising results ${ }^{23}$. As a screening tool, it can be used as the first diagnostic method and patients that show altered sensation can then be referred to undertake a full battery of $\underline{\mathrm{QST}}$ to better document their condition. This may possibly save 
time and costs and allow screening before and after surgery, especially in procedures with possible risk of nerve damage. However, in order to be able to recommend this test in the future, it is important to assess its agreement with QST.

The aim of this study was therefore to assess the level of agreement between quantitative and qualitative sensory testing of experimental modulation of facial sensitivity with capsaicin in a randomized placebo-controlled cross-over manner in healthy individuals. We hypothesized that the agreement between QualST and QST was sufficient for QualST to be used as a screening tool in the clinical setting. Another aim was to further explore the agreement between QST and QualST in assessing the intraoral somatosensory function in clinical atypical odontalgia (AO) patients.

\section{Materials and Methods}

Eighteen healthy pain-free adults ( $>18$ years) were included in the study (10 women, 8 men, mean age $( \pm \mathrm{SD}) 30.9 \pm 5.8$ years). The participants were recruited through advertisements at Aarhus University and the local community. The inclusion criteria were: $>18$ years old. Exclusion criteria were: known systemic problems, current or previous radiotherapy or chemotherapy, intake of any type of analgesics in the last 24 hours prior to the study, presence of any orofacial pain conditions, presence of self-reported psychiatric or personality disorders. The sample size was based on knowledge from earlier studies ${ }^{17-19,21,22}$ on the variance of QST measures performed in the orofacial region taking into account the paired study design.

The study was performed in accordance with the Helsinki Declaration II. The study protocol was approved by the ethics committee of Central Denmark Region. Full explanation of all procedures was given to all participants, after which they signed a written informed consent.

Data from an earlier study using QST and QualST in the chronic intraoral pain condition AO patients were further explored ${ }^{23}$. The $27 \mathrm{AO}$ patients consist of 23 women and 4 men with a mean age $( \pm \mathrm{SD})$ of $63 \pm 14$ years $)$.

Study Design 
The study was designed as a randomized, placebo-controlled cross-over study. All healthy participants were evaluated in two sessions by the same male examiner (one session per tested condition). The tested conditions were: Topical capsaicin and placebo. The tested areas were the right or left infraorbital region of the participants. The condition as well as test side were randomly chosen for each participant. Participants were blinded to the condition being tested. Each test was separated by at least three days. Qualitative sensory testing (QualST) as well as Quantitative sensory testing (QST) were performed on the selected side before and after application in each session.

\section{Topical application}

The concentration of capsaicin and the time-period of application were chosen based on the results of earlier studies ${ }^{25,28}$. The topical application of capsaicin was used to provoke mild to moderate pain that lasted throughout the test period. In this study, 0.1\% capsaicin cream (Capzasin-HP. Chattem, Inc, USA) was used to provoke mild to moderate pain and Mepore Pro Plaster (Mölnlycke Healthcare $\mathrm{AB}$, Göteborg, Sweden) was used as the placebo. A thin layer of capsaicin applied on a $4 \times 4-\mathrm{cm}$ Mepore pro plaster, or a $4 \times 4-\mathrm{cm}$ Mepore pro plaster (placebo), was applied on the infraorbital region of participants. Participants were asked to score the perceived pain intensity every minute during the 5 min of application using a 0-10 Numerical rating scale (NRS) $(0=$ 'no pain', $10=$ 'most pain imaginable').

\section{Measurement process}

Extraoral qualitative sensory testing The applied QualST technique has been previously described in detail $^{23}$. In short, the sensitivity to touch, cold, and pinprick stimuli were evaluated on the test site compared with the contralateral site. The stimuli were applied to the contralateral site first, followed by the test site (application side), and always in the same order: (1) touch, (2) cold, and (3) pinprick ${ }^{23}$. The touch stimulus was applied with a Q-tip in a single stroke over 1 to $2 \mathrm{~cm}$ of skin ${ }^{23}$. The cold stimulus was applied with a stainless steel dental spatula (kept cool in ice water, approximately $0^{\circ} \mathrm{C}$ ) for 1 to $2 \mathrm{~s}^{23}$. The pinprick stimulus was applied with a dental examination probe with moderate force on the skin for 1 to $2 \mathrm{~s}^{23}$. Participants were asked to report hypersensitivity, hyposensitivity, or 
normosensitivity to touch, cold, and painful stimuli on the test site compared with the control contralateral site ${ }^{22,23}$.

Extraoral quantitative sensory testing A standardized quantitative somatosensory examination, according to the protocol of the DFNS for extraoral application, was used ${ }^{17,29}$. Briefly, the quantitative somatosensory examination was comprised of 13 test parameters and investigated the following sensory functions in the following sequence: The tests for thermal sensation were performed using a PATHWAY (MEDOC, Ramat Yishai, Israel) thermal sensory testing device. Cold detection threshold (CDT) and warmth detection threshold (WDT) were measured first. The number of paradoxical heat sensations (PHS) was determined during the thermal sensory limen (TSL) procedure, followed by cold pain threshold (CPT) and heat pain threshold (HPT) ${ }^{17}$. The baseline temperature was $32^{\circ} \mathrm{C}$, and all thresholds were obtained with ramped stimuli $\left(1^{\circ} \mathrm{C} / \mathrm{s}\right)$ that were terminated when the participant pressed a button ${ }^{17}$. Cut-off temperatures were 0 and $50^{\circ} \mathrm{C}$, and the contact area of the extra-oral thermode was $9.0 \mathrm{~cm}^{2}{ }^{17}$. During the experiment, the participants were discouraged from looking at the computer screen.

Mechanical detection thresholds (MDTs) were measured using a standardized set of modified von Frey filaments (OptiHair; MARSTOCK Nervtest, Marburg, Germany), which exert forces between 0.25 and $512 \mathrm{mN}^{17}$. The contact area of the von Frey hairs were rounded tips, $0.5 \mathrm{~mm}$ in diameter, to avoid sharp edges that would facilitate nociceptor activation ${ }^{17,21}$. The filament was applied perpendicular to the test site, and the pressure was slowly increased until the filament began to bend ${ }^{18}$. The time needed to bend was standardized to about $1-2 \mathrm{~s}$, and the stimulus was maintained for $1-2 \mathrm{~s}^{17,21}$. The final threshold was the geometric mean of five series of ascending and descending stimulus intensities, where the participant was asked to indicate each time a stimulus was perceived 17,19 .

Mechanical pain thresholds (MPT) were measured using custom-made weighted pinprick stimulators (Aarhus University, Aarhus, Denmark) with fixed-stimulus intensities between 8 and 512 $\mathrm{mN}^{17,22}$. The final threshold of painful pricking or stinging sensation was the geometric mean of five series of ascending and descending stimulus intensities ${ }^{17,19}$. Mechanical pain sensitivity (MPS) was 
tested using the same weighted pinprick stimuli as for MPT ${ }^{17}$. In addition, dynamic mechanical allodynia (ALL) were tested using three light tactile stimulators: a cotton wisp $(\sim 3 \mathrm{mN})$, a cottonwool tip $(\sim 100 \mathrm{mN})$, and a Somedic brush $(\sim 200-400 \mathrm{mN}){ }^{17,22}$. Each of the seven intensities of pinprick and of the three intensities of light stroking was applied five times in a balanced sequence and the subjects were asked to give a pain rating for each stimulus on a $0-100$ numerical rating scale (from $0=$ 'no pain' to $100=$ 'most pain imaginable'). The MPS was calculated as the geometric mean of all pain ratings for pinprick stimuli, and allodynia was calculated as the geometric mean of all pain ratings for light touch stimuli ${ }^{17,21}$.

The wind-up ratio (WUR) was evaluated using a single pinprick stimulus and 10 pinprick stimuli with the same force, repeated at a rate of $1 \mathrm{~Hz}$ and kept constant using a metronome (MA-30; Korg, New York, USA) ${ }^{17}$. Three single pinprick stimuli were alternated with three series of 10 repeated stimuli. The mean pain rating of the series was then divided by the mean pain rating of single stimuli (train/single pinprick) to calculate the WUR ${ }^{17,21}$.

The vibration detection threshold (VDT) test was performed using a Rydel-Seiffer tuning fork $(64 \mathrm{~Hz}, 8 / 8$ scale $){ }^{17}$ that was set in motion and placed in contact with the zygomatic arch of maxilla. The VDT was determined as a disappearance threshold on the $8 / 8$ scale with three stimulus repetitions $17,21$.

The pressure pain threshold (PPT) was measured using a digital pressure algometer (SOMEDIC Algometer, SOMEDIC Sales, Hörby, Sweden) with a rubber-coated tip of $1 \mathrm{~cm}$ in diameter. During the test, pressure was increased at a rate of $50 \mathrm{kPa} / \mathrm{s})^{17,21}$. At the first painful sensation the subjects pressed a button to interrupt stimulation. The PPT was determined as the mean of three recordings ${ }^{17,21}$.

\section{Statistical analyses}

All absolute $\underline{\mathrm{QST}}$ scores are presented as means \pm standard deviation (SD). All QST data, except for PHS, CPT, HPT, and VDT, were logarithmically transformed before statistical analysis ${ }^{17}$. The mean and SD of all QST parameters of all healthy subjects at baseline was calculated and served as the reference for the Z-transformed QST parameters obtained after application. The QST parameters of 
each subject were transformed into a Z-score using the following equation: Z-score $=\left(\right.$ Value $_{\text {subject }}-$ $\mathrm{Mean}_{\text {baseline}}$ )/ $\mathrm{SD}_{\text {baseline }}$ (negative Z-score: loss of sensory function, positive Z-score: gain in sensory function) ${ }^{17,22}$. Z-scores of $>1.96$ and $<-1.96$ indicate somatosensory sensitivity outside the $95 \%$ CI of the baseline sensitivity of the healthy subjects ${ }^{17}$. P values less than 0.05 were considered statistically significant.

The Loss-Gain score was used to combine a score of somatosensory loss of function (L0, L1, L2, or L3) with a score of somatosensory gain of function (G0, G1, G2, or G3) ${ }^{30,31}$. The number after the ' $\mathrm{L}$ ' or ' $\mathrm{G}$ ' was used to indicate, whether the somatosensory abnormality was related to the thermal modalities alone (1), mechanical modalities alone (2) or mixed (3) (thermal and mechanical) ${ }^{22}$. L0 and G0 were used to indicate no loss or gain of somatosensory function, respectively. L1 indicated isolated loss of small fiber function (if thermal detection thresholds (CDT or WDT) is abnormal); L2 indicated isolated loss of large fiber function (if mechanical detection thresholds (MDT or VDT) is abnormal), and L3 indicated mixed loss of function (if loss of both small and large fiber function) ${ }^{20,22}$. For somatosensory gain, G1 indicated thermal hyperalgesia, if gain of function in cold or heat pain thresholds (CPT or HPT) was found. G2 indicated mechanical hyperalgesia, if gain of function was detected for mechanical pain threshold (MPT), mechanical pain sensitivity (MPS), pressure pain threshold (PPT) or if the dynamic mechanical allodynia (DMA) score exceeded 0. G3 indicating mixed gain of somatosensory function was recorded in individuals with gain of both thermal and mechanical somatosensory function ${ }^{20,22}$.

To assess the agreement between QST and QualST, the Z-scores were categorized as normal, when Z-scores were between 1.96 and -1.96, hypersensitive, when the Z-scores were $>1.96$ or hyposensitive, when the Z-scores were $<-1.96$. Using the Loss-Gain coding ${ }^{30,32}$, the absolute agreement between QualST (3 modalities) and QST (LossGain codes) was calculated as the proportion of the group where hyposensitivity to touch in QualST was in agreement with a L2 or L3 score (both including tactile loss) ${ }^{22}$. Similar proportions were computed for the proportions of the groups showing agreement between hypersensitivity to touch (QualST) and G2 or G3 score; hyposensitivity to cold (QualST) and L1 or L3 score; hypersensitivity to cold (QualST) and G1 or G3 score; hypersensitivity to pinprick (QualST) and G2 or G3 score ${ }^{22}$. 
In addition, the sensitivity and specificity of QualST to detect hyper- and hyposensitivity was evaluated using QST as a benchmark. Kappa statistic was not performed in the present study due to its shortcomings such as dependence on marginal sum as well as prevalence of examining condition ${ }^{33,34}$. These shortcomings make kappa to have a wide confidence interval that may include anything from good to poor agreement ${ }^{34}$. Furthermore, a supplementary explorative analysis of data from a previous study was done in order to further investigate the agreement between QST and QualST in assessing the intraoral sensory function in clinical atypical odontalgia (AO) patients ${ }^{22}$.

NRS pain scores for capsaicin were compared between baseline up till 5 minute post application period with a one-way ANOVA test.

The QST parameters were analyzed with two-way repeated measurements (RM) analyses of variance (ANOVAs) with condition (capsaicin and placebo) and time (before and after application) as factors. Tukey honestly significant difference (HSD) tests with correction for multiple comparisons were used for post hoc analysis when appropriate. All tests were carried out using the STATISTICA, v 12 (StatSoft Inc., USA) Statistical Package.

\section{Results}

\section{QST /QualST findings}

The healthy participants' responses to test conditions as recorded by QST and QualST are presented in Table 1. In two-third of healthy participants, QST recorded normal sensation to touch and cold after the application of capsaicin, while QualST recorded only one-third of the participants as having normal sensation after capsaicin application.

\section{Agreement between QST and QualST (healthy participants) \\ Percentage agreement}

The absolute percentage agreement between corresponding measures from QST and the three modalities of QualST is presented in Table 2. The percentage agreement of touch hyposensitivity or hypersensitivity for QualST and mechanical loss (L2 or L3) or gain (G2 or G3) from QST was 61\% 
(capsaicin session) and 89\% (Placebo), while absolute agreement between cold hyposensitivity or hypersensitivity from QualST and QST (L1 or L2) or (G1 or G3) was 50\% (Capsaicin) and 89\% (Placebo). The percentage absolute agreement of hypersensitivity to pinprick for QualST and mechanical gain (G2 or G3) from QST was 72\% (Capsaicin) and 94\% (placebo). Very little absolute disagreement between QualST and QST was recorded in the capsaicin session in touch and cold modalities (one subject each). In both cases QST indicated hypersensitivity while QualST indicated hyposensitivity.

\section{Changes in classification from QST to QualST (healthy participants)}

Using QST as a benchmark, we compared the corresponding score of QualST for the condition and test modality used to assess facial sensitivity. Figure 1 shows the classification distribution between QST and QualST in healthy participants. There was better agreement after placebo application than after capsaicin for all test modalities.

\section{Sensitivity and specificity (healthy participants)}

The sensitivity and specificity of corresponding measures from QST and the three modalities of QualST in healthy participants are presented in Table 3 . The sensitivity was calculated as the probability of a modality being classified as hyper- or hyposensitive with QualST when the subject was classified as hyper- or hyposensitive with QST. The sensitivity of QualST modalities to detect hyposensitivity ranged from 0.6 to 1.0 with cold modalities presenting the lowest sensitivity after application of capsaicin. While the specificity to detect hyposensitivity ranged from 0.5 to 1.0 with the lowest specificity value seen in touch modality after application of capsaicin Table 3 .

Since no subject was classified as hyper- or hyposensitive with QST or QualST, no estimate (“-“) for the sensitivity was obtained (Table 3). No subjects were classified as hypersensitive in conditions such as capsaicin and placebo. All subjects classified as hypersensitive to pinprick by QualST was also classified as hypersensitive by QST giving a sensitivity of 1.0. The specificity to detect hypersensitivity ranged from 0.7 to 1.0 (Table 3 ). 
Agreement between QualST and QST in AO patients

Changes in classification from QST to QualST (AO patients) The percentage agreement between corresponding measures of QST and QualST in AO patients has been published before ${ }^{23}$. The classification distribution between QST and QualST in AO patients when QST is used as the benchmark is shown in Fig. 2.

\section{Pain evoked by topical application}

All healthy participants scored application of capsaicin as painful. The mean peak pain induced by capsaicin was $8.0 \pm 2.0$, after $5 \mathrm{~min}$. The mean NRS score after capsaicin application increased with time (ANOVA: $\mathrm{F}=116.35, \mathrm{p}=0.001)($ Fig. 3). No participant scored placebo as painful at any time point (Fig. 3).

\section{QST findings_(healthy participants)}

There were no significant main effects of session (ANOVA: $F=1.96, p=0.179$ ) or time (ANOVA: $F$ $=7.39, \mathrm{p}=0.014)$ on CDT scores but there was a statistically significant interaction between session and time (ANOVA: $\mathrm{F}=13.24, \mathrm{p}=0.002$ ). The post hoc analysis showed that application of capsaicin induced a significant decrease in CDT (sensory loss) compared with baseline (Tukey: $p=0.001$ ) and placebo (Tukey: $\mathrm{p}=0.019)$ (Fig. 4).

There were main effects of session (ANOVA: $\mathrm{F}=11.56, \mathrm{p}=0.003$ ) and time (ANOVA: $\mathrm{F}=$ $11.91, \mathrm{p}<0.001)$, but without significant interactions, for the WDT. The WDT scores in the capsaicin session were significantly lower (sensory gain) compared with the placebo session (Tukey: p < 0.003). The post hoc analysis of the time effect indicated higher WDTs after applications compared with before applications (Tukey: $\mathrm{p}<0.001$ ) (Fig. 4).

There was no significant main effect of session (ANOVA: $F=0.74, p=0.401$ ) on TSL values. However, there was a significant main effect of time (ANOVA: $F=46.09, p<0.001$ ). The post hoc analysis showed significantly higher TSL values (decreased sensitivity) after application compared to the pre-application values $(\mathrm{P}<0.001)$ (Fig. 4). 
There was a main effect of session (ANOVA: $\mathrm{F}=7.13, \mathrm{p}=0.016)$ and time $(\mathrm{F}=5.98, \mathrm{p}=$ 0.026) on CPT. Also, there was a statistically significant interaction between session and time (ANOVA: $\mathrm{F}=1129, \mathrm{p}<0.001$ ). The post hoc analysis of the session effect showed that CPT values during the capsaicin session were significantly lower (sensory loss) than in the placebo session (Tukey: $\mathrm{p}<0.016$ ). The post hoc analysis of the interaction between session and time indicated that post application values of CPT during the capsaicin session were lower than at baseline (Tukey: $\mathrm{p}<$ 0.001 ) and in comparison with post application values in the placebo sessions (Tukey: $p<0.001$ ) (Fig. 4).

There were main effects of session (ANOVA: $\mathrm{F}=17.01, \mathrm{p}<0.001)$ and time (ANOVA: $\mathrm{F}=$ 20.86, $\mathrm{p}<0.001)$ on HPT. Also, there was a statistically significant interaction between session and time (ANOVA: $\mathrm{F}=19.90, \mathrm{p}<0.001$ ). The post hoc analysis of the main session effect indicated that the HPT values during the capsaicin session were significantly lower (sensory gain) compared with the placebo session (Tukey: $\mathrm{p}<0.001$ ). The post hoc analysis of the main effect of time indicated slight decreases in HPT values post application compared with baseline (Tukey: $p<0.001$ ). The post hoc analysis of the interaction between session and time indicated a significant decrease in HPT after capsaicin application compared with baseline (Tukey: $\mathrm{p}<0.001$ ) and compared with after application of placebo (Tukey: $\mathrm{p}<0.0001$ ) (Fig. 4).

There was a tendency towards a significant main effect of session (ANOVA: $\mathrm{F}=4.38, \mathrm{p}=$ 0.052) on MDT values. However, there was a significant main effect of time (ANOVA: $F=17.64, p$ $<0.001)$. The post hoc analysis of the time effect indicated higher MDTs after applications compared with baseline (Tukey: $\mathrm{p}<0.001$ ) (Fig. 5).

There was no significant main effects of session (ANOVA: $\mathrm{F}=0.88, \mathrm{p}=0.362$ ) or time (ANOVA: $\mathrm{F}=0.77, \mathrm{p}<0.392$ ) on MPT. Also, there was no statistically significant interaction between session and time (ANOVA: $\mathrm{F}=1.00, \mathrm{p}=0.330$ ) (Fig. 5).

There was a significant main effect of session (ANOVA: $F=4.72, p=0.044$ ) but no main effect of time (ANOVA: $\mathrm{F}=1.22, \mathrm{p}<0.284$ ) on MPS values. The post hoc analysis of the session effect indicated higher MPS values (sensory gain) in the capsaicin session compared with placebo (Tukey: $\mathrm{p}=0.044)$ (Fig. 5). 
There was no significant main effects of session (ANOVA: $F=0.55, p=0.468$ ) but a tendency towards a significant main effect of time (ANOVA: $F=4.16, p=0.057$ ) on WUR. Also, there was no statistically significant interaction between session and time (ANOVA: $F=0.67, p=$ 0.423) (Fig. 5).

There was no significant main effects of session (ANOVA: $\mathrm{F}=2.51, \mathrm{p}=0.131$ ) or time (ANOVA: $\mathrm{F}=1.27, \mathrm{p}=0.275$ ) on VDT scores but there was a statistically significant interaction between session and time (ANOVA: $\mathrm{F}=9.58, \mathrm{p}=0.006$ ). The post hoc analysis of the interaction between session and time indicated significantly lower values of VDT after application of capsaicin compared with baseline (Tukey: $\mathrm{p}<0.027$ ).

There was no significant main effect of session (ANOVA: $F=0.30, p=0.587$ ) or time (ANOVA: $\mathrm{F}=3.83, \mathrm{p}=0.067$ ) on PPT scores but there was a statistically significant interaction between session and time (ANOVA: $\mathrm{F}=7.04, \mathrm{p}=0.017$ ). The post hoc analysis of the interaction indicated that application of capsaicin induced a significant increase in PPT (sensory loss) compared with baseline (Tukey: $\mathrm{p}=0.033$ ) (Fig. 5).

\section{Discussion}

This study assessed the level of agreement between quantitative and qualitative sensory testing assessing the modulation of facial sensitivity by capsaicin and placebo. The test conditions simulated some observed orofacial somatosensory alterations seen after for example after oral surgery. The study also reported on the degree of agreement between quantitative and qualitative sensory testing in patients with a chronic primary intraoral pain condition, i.e., atypical odontolgia.

\section{Agreement between QST and QualST}

A good agreement was observed between quantitative and qualitative sensory testing in healthy participants with no alterations in facial sensation (placebo) with agreement ranging from $89 \%$ to $94 \%$ of the tested participants. The least agreement was seen after capsaicin application in all test modalities with agreement ranging from $50 \%$ to $72 \%$. For most conditions the agreement between QST and QualST was high for the sensitivity to pinprick (72 - 94\%) while the least agreement was 
observed with sensitivity to cold (Table 2). This finding is in agreement with a previous study where good agreement was found between QST and QualST regarding thermal and mechanical modalities in healthy subjects ${ }^{22}$.

QualST seemed to detect more somatosensory anomaly (hyper/hypo) in all sessions/conditions than QST both in healthy participant and AO patients (Tables $1{ }^{23}$ ). This could either mean that QualST tended to induce more false positive responses, or it could mean that the sensitivity of QST is low due to the wide range of normal responses. Since no gold standard is available, this issue is difficult to settle. However, future studies in clinical patient populations with a definite diagnosis of neuropathic pain could be used to observe the degree of agreement between QST and QualST in the patients with an electrophysiological or imaging test documenting a nerve lesion.

Changes in classification between QST and QualST

Fig. 1 shows the classification distribution between QST and QualST in healthy participants. The best agreement was seen in placebo, which indicates that QualST was able to detect normal sensitivity as normal in about $90 \%$ of cases. The high level of specificity shown by QualST reflects the probability that the QualST will be negative among those who, in fact, do not have somatosensory anomaly. The commonest misclassification observed was the classification of normal according to $\underline{\text { QST }}$ as hyposensitivity by QualST. After capsaicin application, participants classified as normal using QST were misclassified as hyper- or hyposensitive using QualST especially for cold and pinprick. A similar trend was observed in the further exploration of earlier reported data on AO patients, where patients classified as normal using QST were misclassified as hypersensitive and in few patients as hyposensitive using QualST (Figure 2). Some patients scored by QST as hypersensitive were scored by QualST as hyposensitive especially in touch and pinprick. This misclassification may result from differences in the psychophysical method and responses obtained from the patients between the two systems (QST and QualST). In QualST, patients were asked to evaluate their sensitivity to the different modalities by comparing one side to the other ${ }^{23}$, whereas with QST somatosensory sensitivity was evaluated based on the means and SDs of the reference ${ }^{17,18}$. 
Although QualST was able to categorize most healthy participants without experimental modulation of facial sensitivity as having normal sensory function, its inconsistency in assessing facial sensitivity after experimental modulation in healthy participants or in clinical AO patients ${ }^{23}$ should be taken into consideration when used for patient screening.

In all 18 healthy subjects, topical application of capsaicin to the infraorbital region caused a severe level of pain. The pain observed in this study was higher than what was reported in a previous study, where capsaicin was applied to the gingiva ${ }^{19}$. The observed high level of pain in the infraorbital region in comparison with gingiva may be due to differences in the C-fiber densities between the tissues ${ }^{25,35}$

In the present study, the application of capsaicin increased CDT and CPT (decreased sensitivity) and reduced HPT (increased sensitivity), which is in accordance with previous studies 19,36. These thermal threshold differences were statistically significant in the direct comparison of data obtained before and after application. In terms of the mechanical stimuli, topical application of capsaicin induced reduced sensitivity to painful mechanical stimuli (the PPT increased), which is in accordance with what was obtained with intraoral application, where topical application of capsaicin to the attached gingiva induced decreased mechanical sensitivity ${ }^{19}$. However, in contrast, other studies on the skin have shown increase in mechanical sensitivity after topical application or intradermal injection of capsaicin in human subjects ${ }^{37-39}$ It could be considered a limitation of the present study that capsaicin application may cause the self-unblinding due to its burning sensation. However, but we do not believe that this influences the agreement between QST and QualST measurements.

Some of the observed changes in somatosensory sensitivity after topical applications were not specific to the application of capsaicin but also occurred after placebo, suggesting that the altered sensitivity to some test stimuli (WDT, TSL and MDT) after application could be speculated to be sensitizations or adaptations to the stimuli rather than changes induced by application ${ }^{19,40}$. This fact stresses the need for a control condition in studies such as this. 
The full QST somatosensory Z-score profile after application of capsaicin and placebo were also reported in the present study. The mean and SD of the QST baseline values (before application) were used as the reference values to compute the Z-scores as done in previous studies ${ }^{19,32,41}$. In the present study, inspection of the individual Z-scores demonstrated decreased sensitivity towards nonpainful cold, warmth and tactile stimuli and increased sensitivity to pinprick and vibratory stimuli as well as increased wind-up after application of capsaicin (Fig. 6).

However, the other findings observed from the direct comparison of values obtained before and after application of the test substances (Fig. 4 \& 5) could not be identified in the Z- scores and the somatosensory profiles (Fig. 6). This may be due to the natural variation in values that were used to create the Z-scores. Thus, the Z-score transformation may only be able to illustrate the most robust findings because minor, but still significant, group differences detected in the statistical comparison of absolute values were not represented as mean Z-scores outside $95 \%$ CI of the baseline values ${ }^{36}$.

In summary, we observed and confirmed that a standardized QST battery and Z-score-based somatosensory profiling indicated that topical application of capsaicin may be considered as an effective surrogate model of extra-oral pain with concomitant somatosensory disturbances.

\section{Conclusion}

In conclusion, the study showed a good level of agreement ( $89 \%$ to $94 \%$ ) between quantitative and qualitative sensory testing in participants with no alterations in facial sensation (placebo). This result showed that QualST may be used as a screening tool in the clinical setting, especially to show that subjects have normal sensory function. However, in case of experimental modulation of facial somatosensory sensitivity as well as in patients with chronic orofacial pain (AO patients) agreement was less pronounced between quantitative and qualitative sensory testing. This has clinical implications for the interpretation of QualST as a screening tool in patients with somatosensory changes and this should be taken into consideration when it is used in the clinical settings. 


\section{Acknowledgment}

The authors report no conflicts of interest related to this study. Agbaje Jimoh Olubanwo is a postdoctoral researcher of the Fund for Scientific Research (FWO-Vlaanderen).

\section{Figure Legends}

Figure 1: Change in classification from quantitative sensory testing (QST) to qualitative sensory testing (QualST) in healthy participants. 0: normal; 1: hypersensitivity; 2: hyposensitivity.

Figure 2: Change in classification from quantitative sensory testing (QST) to qualitative sensory testing (QualST) in AO patients. 0: normal; 1: hypersensitivity; 2: hyposensitivity.

Figure 3: Healthy participants-reported Numerical Rating Scale (NRS) pain scores \pm (SEM) after the topical application of capsaicin and placebo on the infra-orbital region. The results represent mean values $(\mathrm{n}=18)$ obtained during the 5 min recording period.

Figure 4 \& 5: Mean $( \pm \mathrm{SD})$ of quantitative sensory testing $(\underline{\mathrm{QST}})$ parameters for tested condition before and after application in healthy participants.

Figure 6: Individual Z-score-based quantitative sensory testing (QST) profiles from the infraorbital region after the application of capsaicin and placebo in healthy participants and the averaged Zscores $(C)(n=18)$. The grey area $(-1.96<\mathrm{z}<1.96)$ is the normal range based on the healthy reference. CDT: cold detection threshold; WDT: warmth detection; TSL: thermal sensory limen; CPT: cold pain threshold; HPT: heat pain threshold; MDT: mechanical detection threshold; MPT: 
mechanical pain threshold; MPS: mechanical pain sensitivity; WUR: wind-up ratio; VDT: vibration detection threshold; PPT: pressure pain threshold.

\author{
Reference List
}

(1) Wijbenga JG, Verlinden CR, Jansma J, Becking AG. Stegenga B. Long-lasting neurosensory disturbance following advancement of the retrognathic mandible: distraction osteogenesis versus bilateral sagittal split osteotomy. Int $J$ Oral Maxillofac Surg 2009;38:719-725.

(2) Yamauchi K, Takahashi T, Kaneuji T, Nogami S, Yamamoto N, Miyamoto I et al. Risk factors for neurosensory disturbance after bilateral sagittal split osteotomy based on position of mandibular canal and morphology of mandibular angle. J Oral Maxillofac Surg 2012;70:401-406.

(3) Yoshioka I, Tanaka T, Khanal A, Habu M, Kito S, Kodama M et al. Relationship between inferior alveolar nerve canal position at mandibular second molar in patients with prognathism and possible occurrence of neurosensory disturbance after sagittal split ramus osteotomy. J Oral Maxillofac Surg 2010;68:3022-3027.

(4) Phillips C, Essick G. Inferior alveolar nerve injury following orthognathic surgery: a review of assessment issues. J Oral Rehabil 2011;38:547-554.

(5) Politis C, Sun Y, Lambrichts I. Agbaje JO. Self-reported hypoesthesia of the lower lip after sagittal split osteotomy. Int J Oral Maxillofac Surg 2013;42:823-829. 
(6) Politis C, Lambrichts I. Agbaje JO. Neuropathic pain after orthognathic surgery. Oral Surg Oral Med Oral Pathol Oral Radiol 2014;117:e102-e107.

(7) Renton T. Prevention of iatrogenic inferior alveolar nerve injuries in relation to dental procedures. Dent Update 2010;37:350-6, 358.

(8) D'Agostino A, Trevisiol L, Gugole F, Bondi V. Nocini PF. Complications of orthognathic surgery: the inferior alveolar nerve. J Craniofac Surg 2010;21:1189-1195.

(9) Al-Bishri A, Rosenquist J. Sunzel B. On neurosensory disturbance after sagittal split osteotomy. J Oral Maxillofac Surg 2004;62:1472-1476.

(10) Leira JI, Gilhuus-Moe OT. Sensory impairment following sagittal split osteotomy for correction of mandibular retrognathism. Int J Adult Orthodon Orthognath Surg 1991;6:161167.

(11) Smith JG, Elias LA, Yilmaz Z, Barker S, Shah K, Shah S et al. The psychosocial and affective burden of posttraumatic neuropathy following injuries to the trigeminal nerve. $J$ Orofac Pain 2013;27:293-303.

(12) Donoff RB. Surgical management of inferior alveolar nerve injuries (Part I): The case for early repair. J Oral Maxillofac Surg 1995;53:1327-1329.

(13) Pogrel MA, Jergensen R, Burgon E. Hulme D. Long-term outcome of trigeminal nerve injuries related to dental treatment. J Oral Maxillofac Surg 2011;69:2284-2288.

(14) Renton T, Yilmaz Z. Profiling of patients presenting with posttraumatic neuropathy of the trigeminal nerve. J Orofac Pain 2011;25:333-344.

(15) Renton T, Yilmaz Z. Managing iatrogenic trigeminal nerve injury: a case series and review of the literature. Int J Oral Maxillofac Surg 2012;41:629-637. 
(16) Zuniga JR, Yates DM. Phillips CL. The presence of neuropathic pain predicts postoperative neuropathic pain following trigeminal nerve repair. J Oral Maxillofac Surg 2014;72:24222427.

(17) Rolke R, Baron R, Maier C, Tolle TR, Treede RD, Beyer A et al. Quantitative sensory testing in the German Research Network on Neuropathic Pain (DFNS): standardized protocol and reference values. Pain 2006;123:231-243.

(18) Baad-Hansen L, Pigg M, Yang G, List T, Svensson P. Drangsholt M. Reliability of intraoral quantitative sensory testing (QST) in patients with atypical odontalgia and healthy controls - a multicentre study. J Oral Rehabil 2015;42:127-135.

(19) Lu S, Baad-Hansen L, List T, Zhang Z. Svensson P. Somatosensory profiling of intra-oral capsaicin and menthol in healthy subjects. Eur J Oral Sci 2013;121:29-35.

(20) Maier C, Baron R, Tolle TR, Binder A, Birbaumer N, Birklein F et al. Quantitative sensory testing in the German Research Network on Neuropathic Pain (DFNS): somatosensory abnormalities in 1236 patients with different neuropathic pain syndromes. Pain 2010;150:439-450.

(21) Pigg M, Baad-Hansen L, Svensson P, Drangsholt M. List T. Reliability of intraoral quantitative sensory testing (QST). Pain 2010;148:220-226.

(22) Baad-Hansen L, Pigg M, Ivanovic SE, Faris H, List T, Drangsholt M et al. Intraoral somatosensory abnormalities in patients with atypical odontalgia--a controlled multicenter quantitative sensory testing study. Pain 2013;154:1287-1294.

(23) Baad-Hansen L, Pigg M, Ivanovic SE, Faris H, List T, Drangsholt M et al. Chairside intraoral qualitative somatosensory testing: reliability and comparison between patients with atypical odontalgia and healthy controls. J Orofac Pain 2013;27:165-170.

(24) Eberhard L. [Quantitative Sensory Testing in the facial area: a review]. Z Evid Fortbild Qual Gesundhwes 2013;107:291-296. 
(25) Lu S, Baad-Hansen L, Zhang Z. Svensson P. Reliability of a new technique for intraoral mapping of somatosensory sensitivity. Somatosens Mot Res 2013;30:30-36.

(26) Svensson P, Baad-Hansen L, Pigg M, List T, Eliav E, Ettlin D et al. Guidelines and recommendations for assessment of somatosensory function in oro-facial pain conditions--a taskforce report. J Oral Rehabil 2011;38:366-394.

(27) Poort LJ, van Neck JW. van der Wal KG. Sensory testing of inferior alveolar nerve injuries: a review of methods used in prospective studies. J Oral Maxillofac Surg 2009;67:292-300.

(28) Baad-Hansen L, Jensen TS. Svensson P. A human model of intraoral pain and heat hyperalgesia. J Orofac Pain 2003;17:333-340.

(29) Yekta SS, Smeets R, Stein JM. Ellrich J. Assessment of trigeminal nerve functions by quantitative sensory testing in patients and healthy volunteers. J Oral Maxillofac Surg 2010;68:2437-2451.

(30) Jaaskelainen SK, Teerijoki-Oksa T. Forssell H. Neurophysiologic and quantitative sensory testing in the diagnosis of trigeminal neuropathy and neuropathic pain. Pain 2005;117:349357.

(31) List T, Leijon G. Svensson P. Somatosensory abnormalities in atypical odontalgia: A casecontrol study. Pain 2008;139:333-341.

(32) List T, Leijon G, Helkimo M, Oster A. Svensson P. Effect of local anesthesia on atypical odontalgia--a randomized controlled trial. Pain 2006;122:306-314.

(33) Agbaje JO, Mutsvari T, Lesaffre E. Declerck D. Measurement, analysis and interpretation of examiner reliability in caries experience surveys: some methodological thoughts. Clin Oral Investig 2012;16:117-127.

(34) McHugh ML. Interrater reliability: the kappa statistic. Biochem Med (Zagreb) 2012;22:276-282. 
(35) Schmidt R, Schmelz M, Forster C, Ringkamp M, Torebjork E. Handwerker H. Novel classes of responsive and unresponsive $\mathrm{C}$ nociceptors in human skin. J Neurosci 1995;15:333-341.

(36) Naganawa T, Baad-Hansen L, Ando T. Svensson P. Influence of topical application of capsaicin, menthol and local anesthetics on intraoral somatosensory sensitivity in healthy subjects: temporal and spatial aspects. Exp Brain Res 2015;233:1189-1199.

(37) Kilo S, Schmelz M, Koltzenburg M. Handwerker HO. Different patterns of hyperalgesia induced by experimental inflammation in human skin. Brain 1994;117:385-396.

(38) Koltzenburg M, Lundberg LE. Torebjork HE. Dynamic and static components of mechanical hyperalgesia in human hairy skin. Pain 1992;51:207-219.

(39) Baumann TK, Simone DA, Shain CN. LaMotte RH. Neurogenic hyperalgesia: the search for the primary cutaneous afferent fibers that contribute to capsaicin-induced pain and hyperalgesia. J Neurophysiol 1991;66:212-227.

(40) Shimada A, Castrillon E, Baad-Hansen L, Ghafouri B, Gerdle B, Ernberg M et al. Muscle pain sensitivity after glutamate injection is not modified by systemic administration of monosodium glutamate. $J$ Headache Pain 2015;16:68. doi: 10.1186/s10194-015-0546-0. Epub@2015 Jul 22::68-0546.

(41) Kothari SF, Baad-Hansen L, Andersen K. Svensson P. Neurosensory assessment in patients with total reconstruction of the temporomandibular joint. Int J Oral Maxillofac Surg 2014;43:1096-1103. 
Table 1: Pooled data for healthy participants' response to QST and QualST modalities after application of Capsaicin and placebo. Each number represents the number of participants scoring normal, hyper- or hyposensitivity with QST and QualST

\begin{tabular}{|l|l|l|l|l|l|l|l|l|l|l|l|l|l|l|}
\hline \multicolumn{2}{|c|}{} & \multicolumn{9}{|c|}{ Capsaicin } & \multicolumn{7}{c|}{ Placebo } \\
\hline \multicolumn{2}{|l|}{} & \multicolumn{2}{|c|}{ TOUCH } & \multicolumn{2}{l|}{ COLD } & \multicolumn{2}{l|}{ PINPRICK } & \multicolumn{2}{l|}{ TOUCH } & \multicolumn{2}{l|}{ COLD } & \multicolumn{2}{l|}{ PINPRICK } \\
\hline & & QST & QualST & $\underline{\text { QST }}$ & QualST & $\underline{\text { QST }}$ & QualST & QST & QualST & $\underline{\text { QST }}$ & QualST & QST & QualST \\
\hline Normal & 12 & 6 & 12 & 6 & 16 & 5 & 17 & 18 & 18 & 16 & 18 & 17 \\
\hline Hypersensitivity & 0 & 1 & 0 & 4 & 1 & 5 & 0 & 0 & 0 & 0 & 0 & 1 \\
\hline Hyposensitivity & 6 & 11 & 6 & 8 & 1 & 8 & 1 & 0 & 0 & 2 & 0 & 0 \\
\hline
\end{tabular}

Table 2: Percentage agreement between QST and QualST (Healthy participants)

\begin{tabular}{|l|l|l|l|l|l|l|}
\hline & \multicolumn{3}{|c|}{ Capsaicin } & \multicolumn{3}{c|}{ Placebo } \\
\hline & Touch (\%) & Cold (\%) & Pinprick (\%) & Touch (\%) & Cold (\%) & Pinprick (\%) \\
\hline Absolute Agreement & $11(61)$ & $9(50)$ & $13(72)$ & $16(89)$ & $16(89)$ & $17(94)$ \\
\hline Disagreement & $6(33)$ & $8(44)$ & $5(28)$ & $2(11)$ & $2(11)$ & $1(6)$ \\
\hline Absolute Disagreement & $1(6)$ & $1(6)$ & $0(0)$ & $0(0)$ & $0(0)$ & $0(0)$ \\
\hline
\end{tabular}


Table 3: The Sensitivity, Specificity of QualST given the status of QST (Healthy participants)

\begin{tabular}{|l|l|c|c|c|c|}
\hline & & \multicolumn{2}{|c|}{ Hypersensitivity } & \multicolumn{2}{c|}{ Hyposensitivity } \\
\hline Parameter & \multicolumn{1}{|c|}{ Treatment } & Sensitivity & Specificity & Sensitivity & Specificity \\
\hline \multirow{2}{*}{ Touch } & Capsaicin & - & 0.94 & 0.83 & 0.50 \\
\cline { 2 - 6 } & Placebo & - & 1.00 & - & 1.00 \\
\hline \multirow{2}{*}{ Cold } & Capsaicin & - & 0.78 & 0.67 & 0.67 \\
\cline { 2 - 6 } & Placebo & - & 1.00 & - & 0.89 \\
\hline \multirow{2}{*}{ Pinprick } & Capsaicin & 1.00 & 0.76 & 1.00 & 0.59 \\
\cline { 2 - 6 } & Placebo & - & 0.94 & - & 0.94 \\
\hline
\end{tabular}

The sensitivity is calculated as the probability of being classified as hyper- or hyposensitive with | QualST when the subject was truly hyper- or hyposensitive with QST. When no subject was classified as hyper- or hyposensitive, no estimate ("-“) for the sensitivity was obtained. 
Figure 1: Change in classification from quantitative sensory testing (QST) to qualitative sensory testing (QualST) in healthy participants. 0: normal; 1: hypersensitivity; 2: hyposensitivity.

\section{$127 \times 90 \mathrm{~mm}(300 \times 300 \mathrm{DPI})$}




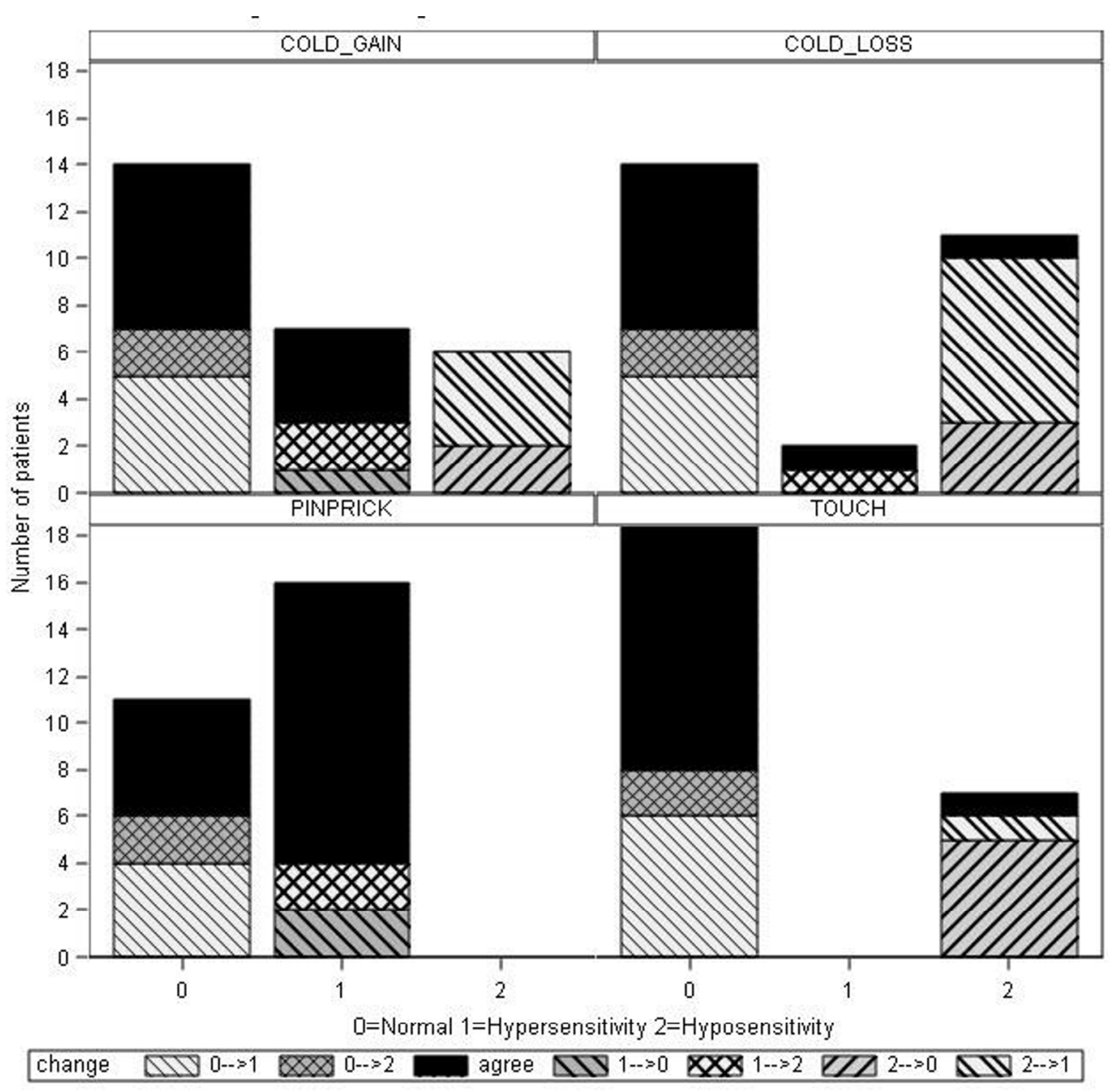

Figure 2: Change in classification from quantitative sensory testing (QST) to qualitative sensory testing (QualST) in AO patients. 0: normal; 1: hypersensitivity; 2: hyposensitivity.

$180 \times 181 \mathrm{~mm}(300 \times 300 \mathrm{DPI})$ 
Figure 3: Healthy participants-reported Numerical Rating Scale (NRS) pain scores \pm (SEM) after the topical application of capsaicin and placebo on the infra-orbital region. The results represent mean values $(n=18)$ obtained during the 5 min recording period.

$$
110 \times 77 \mathrm{~mm}(300 \times 300 \text { DPI })
$$



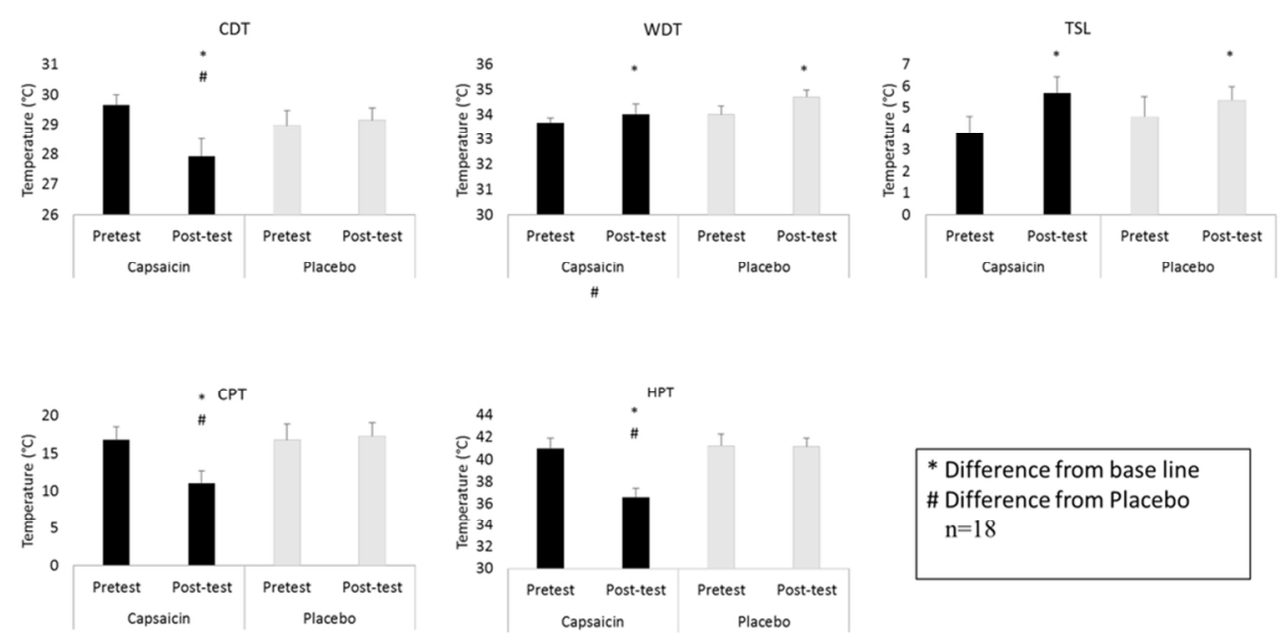

* Difference from base line \# Difference from Placebo $\mathrm{n}=18$

Figure 4: Mean ( $(\mathrm{SD})$ of quantitative sensory testing (QST) parameters for tested condition before and after application in healthy participants.

$88 \times 44 \mathrm{~mm}(300 \times 300 \mathrm{DPI})$ 

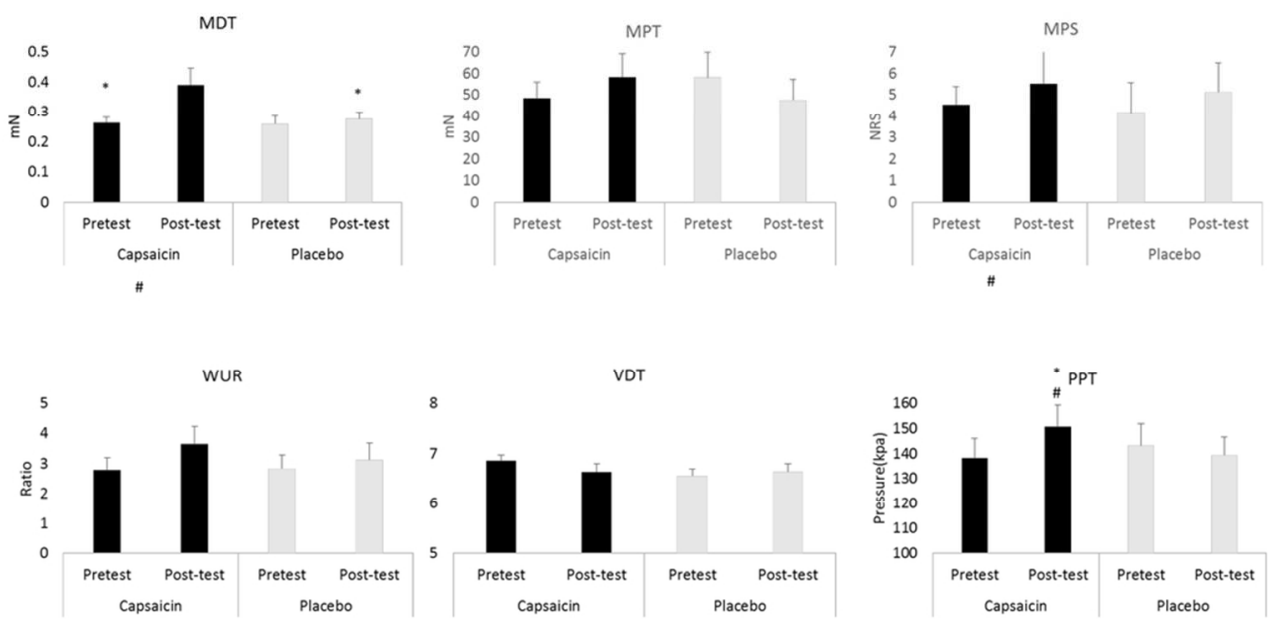

Figure 5: Mean ( $\pm \mathrm{SD}$ ) of quantitative sensory testing (QST) parameters for tested condition before and after application in healthy participants.

$89 \times 44 \mathrm{~mm}(300 \times 300 \mathrm{DPI})$ 
Figure 6: Individual Z-score-based quantitative sensory testing (QST) profiles from the infraorbital region after the application of capsaicin and placebo in healthy participants and the averaged Z-scores (C) ( $n=$ 18). The grey area $(-1.96<z<1.96)$ is the normal range based on the healthy reference. CDT: cold detection threshold; WDT: warmth detection; TSL: thermal sensory limen; CPT: cold pain threshold; HPT: heat pain threshold; MDT: mechanical detection threshold; MPT: mechanical pain threshold; MPS: mechanical pain sensitivity; WUR: wind-up ratio; VDT: vibration detection threshold; PPT: pressure pain threshold.

$100 \times 56 \mathrm{~mm}(300 \times 300$ DPI $)$ 